\title{
Análise da Depressão Geriátrica em uma Comunidade de Maceió - AL
}

Analysis of Geriatric Depression in a Community of Maceió-AL

Análisis de la Depresión Geriátrica en una Comunidad de Maceió-AL

Madson Alan Maximiano-Barreto ${ }^{1}$

Iago Moura Aguiar ${ }^{2}$

André Fernando de Oliveira Fermoseli ${ }^{3}$

\section{Resumo}

Objetivo: Rastrear a ocorrência de depressão geriátrica em idosos de uma comunidade de Maceió - AL. Método: Trata-se de um estudo transversal intencional não-probabilístico realizado com 86 idosos, os quais apresentam idade igual ou superior à 60 anos e são cadastrados na Estratégia de Saúde da Família. Utilizou-se como método de pesquisa um questionário semiestruturado e a Escala de Depressão Geriátrica - EDG versão reduzida. Resultados: Verificou-se que no EDG15 a ocorrência de sintomatologias depressiva é de 46,06\% $(\mathrm{n}=40)$ dos idosos entrevistados, dos quais houve $72,50 \%(\mathrm{n}=56)$ de prevalência em mulheres. Conclusão: Diante dos resultados, percebe-se que os resultados não diferem dos estudos realizados com o mesmo objetivo. Além disso, os fatores sociais são comumente apresentados, no entanto, pouco são as discussões, visto que, são fatores que corroboram para o acometimento da Depressão e de outras psicopatologias.

\section{Descritores: Depressão; Idosos;}

\section{Envelhecimento.}




\section{Abstract}

Objective: Trace the occurrence of geriatric depression in the elderly in a community of Maceió-AL. Method: Is a cross-sectional study non-probabilistic intentional performed with 86 elderly, which feature age greater than or equal to 60 years and are registered in The family health strategy. It was used as a semi-structured questionnaire survey method and the geriatric depression scale GDS-reduced version. It was found that the GDS-15. Results: The occurrence of clinical symptoms depression is $46.06 \%(n=40)$ of the elderly respondents, of which there was $72.50 \%(n=56)$ of prevalence in women. Conclusion: On the results, the results do not differ from studies carried out with the same goal. In addition, the social factors are commonly presented, however, just discussions, are factors that support for the involvement of the depression and other psychopathologies.

\section{Descriptors: Depression; Aged; Aging.}

\section{Resumen}

Objetivo: Trazar que la ocurrencia de depresión geriátrica en el anciano en una comunidad de Maceió - AL.
Método: Es un estudio transversal no probabilístico intencional realizada con 86 personas de edad avanzada, que característica mayor o igual a 60 años de edad y están registradas en La estrategia de salud de la familia. Fue utilizado como un método de encuesta de cuestionario semi-estructurado y la versión reducida de EDG escala de depresión geriátrica. Los resultados: Se encontró que la EDG-15 la ocurrencia de depresión síntomas clínicos es $46.06 \%(n=40)$ de los ancianos encuestados, de los cuales había $72.50 \%(n=56)$ de la prevalencia en las mujeres. Conclusión: En los resultados, los resultados no difieren de estudios realizados con el mismo objetivo. Además, los factores sociales que comúnmente presentan, sin embargo, sólo discusiones, son factores que apoyan la participación de la depresión y otras psicopatologías.

\section{Descriptores: Depresión; Anciano;}

Envejecimento.

\section{Introdução}

O número de idosos no Brasil e no mundo vem aumentando a cada ano. $\mathrm{O}$ aumento da população nos países mais desenvolvidos é um fenômeno que não abarca tantas consequências como nos 
países em desenvolvimento, uma vez que estes não encontram-se preparados para atender a demanda desta população, como é o caso do Brasil ${ }^{(1-2)}$.

Acredita-se que até 2025 o Brasil apresentará um aumento de indivíduos com idade igual ou superior a 60 anos bastante considerável, chegando aos 32 milhões. A saber, a região Sudeste apresenta o maior número de idosos $46 \%$, ficando atrás apenas da região Nordeste com $27 \%$ do total da população brasileira ${ }^{(3)}$.

Considerando o aumento de idosos muito acelerado, concomitante, surge o aumento de doenças físicas e psíquicas. As doenças comumente mais ocorridas nos indivíduos que têm atingido a velhice são Diabetes Mellitus e a Hipertensão Arterial, além dos diversos transtornos que afetam os indivíduos geriátricos ${ }^{(4)}$. Diante de tantas psicopatologias, chama-se à atenção a depressão, uma vez que essa, tem apresentado uma ocorrência negativamente considerável, e principalmente, nos idosos, chegando a afetar a qualidade de vida ${ }^{(2,5)}$.

Levando em consideração as manifestações e gravidade da psicopatologia, em especial à depressão, o número de idosos identificados com sintomatologias depressiva varia de 5\% a $35 \%$. No caso de idosos não institucionalizados esse resultado difere, entre $15 \%$ e $20 \%$. Para esse resultado, leva-se em consideração alguns fatores apresentados na literatura como sexo, escolaridade, nível socioeconômico, condições de saúde e outros ${ }^{(6-7)}$.

A depressão geriátrica traz diversas consequências para o sujeito, dentre estas, a falta de autonomia devido ao nível da gravidade das patologias já pré-existentes no sujeito. Dessa forma, observa-se o aumento dos riscos de morbidade e mortalidade, fazendo com que ocorra um aumento na utilização dos serviços de saúde, no uso de medicamentos, gerando então, comorbidades e abarcando em uma complexidade no diagnóstico e tratamento da depressão ${ }^{(5)}$.

A falta de profissionais da saúde para atender a demanda da população idosa é perceptível, principalmente quando se trata dos idosos que residem nas comunidades. Dado isso, percebe-se um desafio nas mudanças fisiopatológicas e psicossociais que a velhice apresenta. Causando possíveis entraves capazes de implicar na estrutura familiar, nas comunidades e no serviço de saúde ${ }^{(8)}$.

É importante que os profissionais da saúde que se encontram nas Unidades Básicas de Saúde passem a valorizar os sinais e sintomas, uma vez 
que a negligência e não aplicabilidade desta valorização pode gerar o agravo das doenças crônicas, a exclusão social e dificuldade do sujeito a aceitar o tratamento $^{(2)}$.

Considerando que o número de idosos que residem nas comunidades tem se elevado e consequentemente o aumento da população com depressão, dentre a sua maioria, os indivíduos que atingiram a velhice. Dessa forma, esse estudo teve como objetivo rastrear a ocorrência da depressão em idosos que residem em uma comunidade localizada na área urbana de Maceió - AL que apresentam variáveis capazes de possibilitar ao indivíduo o acometimento desta e outras psicopatologias.

\section{Método}

Trata-se de um estudo de natureza quantitativo, descritivo. Os idosos estudados são atendidos em uma Unidade Básica de Saúde da comunidade e cadastrados no Estratégia Saúde da Família. A amostra foi por conveniência composta por 86 idosos que residem na comunidade. A distribuição desses idosos é de 57 mulheres e 29 homens com idade compreendida entre os 60 e 90 anos $(\overline{\mathrm{X}}$ $=68,05 ; \mathrm{DP}=6,7)$. Definiram-se como critérios de inclusão os idosos que tivessem idade igual ou superior a 60 anos e que fossem cadastrados no programa estratégia saúde da família, e como critério de exclusão os idosos que apresentassem qualquer tipo de deficiência que não the permitisse participar de todas as etapas da pesquisa.

$\mathrm{O}$ projeto que originou este artigo foi aprovado pelo Comitê de Ética em Pesquisa (parecer $\mathrm{n}^{\mathrm{o}}$ 1.602.998), seguindo os padrões éticos como rege a Resolução Ética 466/2012.

Para identificação das variáveis sociodemográficas como: idade, estado civil, escolaridade, e outros foi aplicado um questionário semiestruturado desenvolvido pelos próprios pesquisadores.

Para pesquisar a depressão foi escolhida a EDG-15, por ser um instrumento autoaplicável e bastante presente nas pesquisas realizada em idosos de diversos âmbitos (Comunidades, Instituições de Longa Permanência, Clínicas e Hospitais). A Escala de Depressão Geriátrica (EDG) foi desenvolvida em 1983 por Yesavage e colaboradores. $\mathrm{O}$ primeiro instrumento é comporto por 30 questões com respostas dicotômicas $\operatorname{sim} /$ não. No entanto, utilizou-se a EDG-15 versão reduzida, visto que Almeida e Almeida 
(1999) em seu estudo mostraram que a

EDG-15 apresentou boa confiabilidade para identificação de possível acometimento de depressão em idosos.

Inicialmente foi contactado o presidente da comunidade situada em Maceió e os agentes de saúde da Unidade de Básica de Saúde, informando acerca do estudo solicitando autorização para realização da pesquisa. Feito isso, houve uma seleção por parte dos agentes de saúde do programa Estratégia Saúde da Família e em conjunto com os pesquisadores selecionou-seos idosos. Posteriormente, fez-se as visitas nas residências, no período de junho a agosto de 2016 durante todos os dias da semana nos turnos da manhã e tarde.

Apresentou-se aos idosos o objetivo da pesquisa e disponibilizado o Termo de Consentimento Livre e Esclarecido (TCLE) para serem assinados. Os questionários foram aplicados de forma individual, em ambiente disponibilizado pelos próprios participantes em que se sentissem à vontade.

Após o preenchimento de todos os instrumentos realizou-se a análise estatística através do programa Statistica Package for the Social Sciences -SPSS versão 23.0, de maneira descritiva, por frequência relativa e percentual, desvio padrão. Posteriormente utilizou-se também os testes indutivos como o Chi-Quadrado $\left(\chi^{2}\right)$, para demonstrar se existe ou não relação entre variáveis, e o teste de correlação de Pearson para definir se variáveis são diretamente ou inversamente proporcionais.

\section{Resultados}

As características dos idosos que residem na comunidade e que são cadastrados na Estratégia Saúde da Família são apresentadas na tabela 1 . Observa-se a predominância de indivíduos que se autodeclararam pardos, com ensino fundamental incompleto e do sexo feminino. No que concerne aos principais trabalhos apresentados pelos idosos entrevistados, a maioria das mulheres desenvolvem atividade de doméstica, e os homens o serviço de pedreiro. A saber, $16 \%$ dos idosos encontram-se aposentados, 22,09\% recebem Benefício assistencial ao idoso e à pessoa com deficiência (BCP) ou Benefício de Prestação Continuada (LOAS), 5,81\% são pensionistas, no entanto, muitos desses idosos ainda continuam realizando essas e outras atividades. 
Tabela 1. Caracterização dos Idosos em uma Comunidade de Maceió - AL

\begin{tabular}{lcc}
\hline Variável & $\mathbf{n}$ & $\mathbf{\%}$ \\
\hline Sexo & & \\
\hline Masculino & 57 & 66,28 \\
Feminino & 29 & 33,72 \\
\hline Idade & 56 & \\
\hline 60 a 89 anos (Feminino) & 29 & 36,28 \\
60 a 90 anos (Masculino) & & \\
\hline Etnia & 14 & 16,28 \\
\hline Negro & 20 & 23,26 \\
Branco & 3 & 3,49 \\
Amarelo & 49 & 56,98 \\
Parda & & \\
\hline Escolaridade & 33 & 38,37 \\
\hline Analfabeto & 44 & 44,64 \\
Fundamental incompleto & 7 & 13,21 \\
Ensino médio completo & 1 & 1,89 \\
Curso técnico & 1 & 1,89 \\
Educação superior completo & & \\
\hline Atividades remuneradas & 12 & 26,67 \\
\hline Doméstica & 9 & 20,00 \\
Lavadeira & 5 & 11,11 \\
Costureira & 5 & 11,11 \\
Cozinheira & 9 & 20,00 \\
Pedreiro & 3 & 6,67 \\
Motorista & 2 & 4,44 \\
Serviços Gerais & 2 & 4,44 \\
Mecânico & & \\
\hline & & \\
\hline
\end{tabular}

No que concerne a ocorrência de depressão entre os idosos do sexo feminino e masculino a tabela 2 mostra sua distribuição, junto a frequência da depressão entre os idosos. Obteve-se os resultados de $45,35 \%$ para nível de depressão leve a moderado e 1,16\% depressão severa.

Tabela 2 - Ocorrência de Depressão em Idosos de Forma Geral e por Sexo da Comunidade Através do EDG-15

\begin{tabular}{lcc}
\hline Descrição & $\mathbf{n}$ & $\mathbf{\%}$ \\
\hline Com depressão & 40 & 46,51 \\
Sem depressão & 46 & 53,49 \\
Depressão por sexo & & \\
Masculino & 11 & 27,50 \\
Feminino & 29 & 72,50 \\
\hline
\end{tabular}


A tabela 3 exibe a composição da EDG-15 de forma detalhada quanto aos resultados obtidos por cada idoso.
Não se encontrou relação estatísticas entre o acometimento de depressão e sexo entre os pesquisados.

Tabela 3 - Resultados Detalhado de cada Questão da Escala Geriátrica de Depressão (EDG - 15) com Idosos da Comunidade em Maceió - AL

\begin{tabular}{lcccccc}
\hline \multirow{2}{*}{ Descrição } & \multicolumn{2}{c}{ Sim } & \multicolumn{2}{c}{ Não } & \multicolumn{2}{c}{ Total } \\
& $\mathbf{n}$ & $\mathbf{\%}$ & $\mathbf{N}$ & $\mathbf{\%}$ & $\mathbf{n}$ & $\mathbf{\%}$ \\
\hline 01. Está satisfeito (a) com sua vida & 73 & 84,9 & $* 13$ & 15,1 & 86 & 100 \\
02. Diminuiu a maior parte de suas & $* 62$ & 72,1 & 24 & 27,9 & 86 & 100 \\
atividades e interesses & & & & & & \\
03. Sente que a vida está vazia & $* 44$ & 51,2 & 42 & 48,8 & 86 & 100 \\
04. Aborrece-se com frequência & $* 54$ & 62,8 & 32 & 37,2 & 86 & 100 \\
05. Sente-se de bem com a vida na maior & 68 & 79,1 & $* 18$ & 20,9 & 86 & 100 \\
parte do tempo & & & & & & \\
06. Teme que algo ruim possa lhe acontecer & $* 52$ & 60,6 & 34 & 39,5 & 86 & 100 \\
07. Sente-se feliz a maior parte do tempo & 71 & 82,6 & $* 15$ & 17,4 & 86 & 100 \\
08. Sente-se frequentemente desamparado & $* 34$ & 39,5 & 52 & 60,5 & 86 & 100 \\
09. Prefere ficar em casa a sair e fazer coisas & $* 51$ & 59,3 & 35 & 40,7 & 86 & 100 \\
novas & & & & & & \\
10. Acha que tem mais problemas de & $* 31$ & 36 & 55 & 64 & 86 & 100 \\
memória que a maioria & & & & & & \\
11. Acha que é maravilhoso estar vivo agora & 86 & 100 & $* 0$ & 00 & 86 & 100 \\
12. Vale a pena viver como vive agora & 75 & 87,2 & $* 11$ & 12,8 & 86 & 100 \\
13. Sente-se cheio (a) de energia & 64 & 74,4 & $* 22$ & 25,6 & 86 & 100 \\
14. Acha que sua situação tem solução & 68 & 79,1 & $* 18$ & 20.9 & 86 & 100 \\
15. Acha que tem muita gente em situação & $* 70$ & 81,4 & 16 & 18 & 86 & 100 \\
melhor & & & & & & \\
\hline
\end{tabular}

(*) Questões que correspondem a um ponto, utilizadas para somatória negativa no total da escala.

\section{Discussão}

Verifica-se neste estudo a predominância de indivíduos do sexo feminino, que corrobora com os dados de feminização da velhice, que é um fator bastante observado no Brasil, mostrando as mudanças epidemiológicas e de assistência no perfil desses sujeitos. O número de idosas no país é mais alto em comparação com os homens e, essas por sua vez, necessitarão de cuidados maiores, uma vez que tradicionalmente, são também as cuidadoras ${ }^{(11)}$. Visto isso, surge a necessidade de maior preocupação com os cuidados à saúde da mulher ${ }^{(2)}$.

Hoje em dia existem diversas formas e mecanismos de alfabetização, no entanto, segundo os dados do IBGE, os idosos que apresentam alto nível de escolaridade, ainda não se sobrepõem ao número dos que apresentam baixa 
escolaridade $^{(3)}$. No estudo, há predominância de idosos com fundamental incompleto, ou seja, menos de cinco anos do nível de ensino básico.

Acredita-se que a depressão é considerada um problema de saúde pública, visto que, a sua ocorrência é tão alta entre idosos quanto a presença de doenças mais comuns dessa fase da vida, como é o caso da Diabetes Mellitus e Hipertensão Arterial ${ }^{(11-13)}$.

Este estudo traz uma frequência da sintomatologia depressiva entre os idosos de 46,51\% (tabela 3). É importante levar em consideração as circunstâncias de vida as quais esses idosos encontram-se expostos, assim como o seu contexto histórico de vida, que podem estar relacionados com o acometimento da depressão. Chama-se a atenção de algumas características observadas durante as coletas de dados deste estudo, como por exemplo, a falta de saneamento básico e falta de acesso adequado ao posto de saúde da comunidade, o que mostra a mobilidade e não dependência funcional desses sujeitos quando buscam sair de casa.

Assim, idosos que residem em comunidades em comparação com os que estão institucionalizados e/ou hospitalizados, apresentam menor índice de depressão, como mostram as pesquisas realizadas por Porcu et al ${ }^{(11)}$ que encontrou menor prevalência de depressão em idosos investigados residentes em seus domicílios, o que mostra a importância de se considerar as facetas que compõem o ambiente no qual o sujeito encontra-se inserido ${ }^{(14-16)}$.

Outro fator importante é a qualificação profissional. Aqui 83,01\% (tabela 1) dos entrevistados é de baixo nível de escolaridade, o que pode levar a pensar que não tenham desenvolvido atividades cognitivas, com exigências mais elevada, podendo levar a uma acomodação e uma qualidade de vida mais restrita, monótona, sem estimulo, fator que pode estar entre aqueles contribuintes para o acometimento da depressão. Além disto, os aqui pesquisados não apresentam renda salarial capaz de suprir as necessidades básicas de uma família, muito menos, às necessidades da multidimensionalidade que compõem o envelhecimento ${ }^{(17-18)}$.

Nos EDG-15， 46,51\% deles apresentaram sintomatologias depressivas. Observando-se de forma detalhada, 84,9\% dos idosos relataram estar "de bem com a vida", assim como, $79,1 \%$ revelaram se sentir bem na maior parte do tempo e $100 \%$ acharam que vale a pena estar vivo, e sentem-se estar felizes. Considera-se isto, um fator de proteção para o acometimento da depressão, visto que, essa relação de 
felicidade, como descreve Castro-Costa et $\mathrm{al}^{(19)}$ liga-se a satisfação pessoal e ao autocuidado.

O Brasil vem sofrendo mudanças políticas e econômicas, o que pode gerar uma precariedade na manutenção de saúde dos indivíduos que estão envelhecendo e por conseguinte, um aumento dessa e outras patologias. Desta forma, outro aspecto em destaque, são os fatores sociais, uma vez que, esses possivelmente sejam mais influenciadores do que os fatores fisiológicos. Destaca-se a importância do psicólogo dentro da Estratégia Saúde da Família ${ }^{(20)}$, além da necessidade de despertar da atenção dos demais profissionais inseridos nas Unidades Básicas de Saúde da Estratégia Saúde da Família ${ }^{(21,22)}$ para a saúde mental da população, particularmente, aqui dos idosos, com intuito de promover uma atenção que possibilite um envelhecimento de qualidade.

\section{Conclusão}

Os resultados encontrados neste estudo apresentam consonância com outros estudos realizados em distintas localidades do Brasil. Assim como, a presença do acometimento da depressão em indivíduos do sexo feminino em comparação aos idosos do sexo masculino.

Depreende-se, do exposto, a importância de criação de atividades capazes de promover e possibilitar uma maior relação entre os idosos que residem nas comunidades com a sociedade e a todos que encontram-se no mesmo contexto, com intuito de minimizar as consequências ocasionadas pelo abandono, a exclusão e dentre outros fatores o que por sua vez, leva a depressão e a presença de outras psicopatologias.

\section{Referências}

1. Kalache A, Veras RP, Ramos LR. O envelhecimento da população mundial: um desafio novo. Saúde Pública (São Paulo) [periódico na internet]. 1987 [citado 2017 set. 15]; 21(3): 200-10. Disponível em: http://www.repositorio .unifesp.br/handle/11600/217.

2. Teston EF, Carreira L, Marcon SS. Sintomas depressivos em idosos: comparação entre residentes em condomínio específico para idoso e na comunidade. Enfermagem (Brasília) [periódico na internet]. 2014 [citado 2017 set. 22]; 67(3): 450-6. Disponível em: http://www.scielo.br/pdf/reben/v6 7n3/0034-7167-reben-67-03-0450.pdf.

3. Ministério da Saúde (BR). Instituto Brasileiro de Geografia e Estatística. Sobre a condição de saúde dos idosos: indicadores selecionados. Indicadores Sociodemográficos e de Saúde no Brasil. Brasília: Ministério da Saúde; 2009. 
4. Bhattacharjee S, Oh Y, Reiman E and Burke W. Prevalence, patterns, and predictors of depression treatment among community-dwelling elderly individuals with dementia in the United States. Psychiatry (Denmark) [periódico na internet]. 2017 [citado 2017 set. 15]; 25: 803-13. Disponível em: http://www.sciencedirect.com/sci ence/article/pii/S1064748117302518.

5. Djernes JK. Prevalence and predictors of depression in populations of elderly: a review. Psychiatry (Denmark) [periódico na internet]. 2006 [citado 2017 ago. 10]; 113 (5):372-87. Disponível em: http://onli nelibrary.wiley.com/doi/10.1111/j.160 0-0447.2006.00770.x/full.

6. Melo FGM, Nascimento SNF, Freitas MCKN. Prevalência e determinantes de sintomas depressivos em idosos atendidos na atenção primária de saúde. Saúde Pública (São Paulo) [periódico na internet]. 1987 [citado 2017 set. 15]; 21(3): 200-10. Disponível em: http://www.repositorio. unifesp.br/handle/11600/217.

7. Hartmann JASJ, Gomes GC. Depressão em idosos institucionalizados: padrões cognitivos e qualidade de vida. Ciência e. Cognição (Rio de Janeiro) [periódico na internet]. 2016 [citado 2017 set. 11]; 21(1):137-54. Disponível em: http://cienciasecognicao.org/revista/ind ex.php/cec/article/view/1028/pdf_72.

8. Brito MCC, Freitas CASL, De Mesquita KO, Lima GK. Envelhecimento populacional e os desafios para a saúde pública: análise da produção científica. Gerontologia (São Paulo) [periódico na internet]. 2013 [citado 2017 set. 15]; 16(3):16178. Disponível em: https://revistas.pu csp.br/index.php/kairos/article/view/18 552.
9. Yesavage JA, Brink TL, Rose TL, Lum O, Huang V,Adey $M$, et al. Development and validation of ageriatric depression screening scale: a preliminaryreport. J Psychiat Res. [periódico na internet]. 1983 [citado 2017 set. 23]; 17(1):37-49. Disponível em: http://www.sciencedirect.com/scie nce/article/pii/0022395682900334.

10. Almeida OP, Almeida SA. Confiabilidade da versãobrasileira da escala de depressão em geriatria (GDS) versão reduzida. Neuropsiquiatria (São Paulo) [periódico na internet]. 1999 [citado 2017 ago. 03]; 57(2B):421-6. Disponível em: http://www.scielo.br/ pdf/\%0D/anp/v57n2B/1446.pdf.

11. Camarano AA, Kanso S. Envelhecimento da População Brasileira: uma contribuição Demográfica. In: Freitas EV, Py L, organizadores. Tratado de Geriatria e Gerontologia, Rio de Janeiro: Guanabara Koogan; 2016.

12. Schmidt MI, Duncan BB, e Silva $\mathrm{GA}$, et al. Chronic noncommunicable diseases in Brazil: burden and current challenges. Lancet [periódico na internet]. 2011 [citado 2017 set. 15]; 377(9781):1949-61. Disponível em: http://www.sciencedirect.com/science/ article/pii/S0140673611601359

13. Minghelli B, Tomé B, Nunes $\mathrm{C}$, Neves A, Simões C. Comparação dos níveis de ansiedade e depressão entre idosos ativos e sedentários. Psiquiatria (Portugal) [periódico na internet]. 2013 [citado 2017 ago. 25]; 40(2):71-6. Disponível em: https://www.researchg ate.net/profile/Carla_Nunes3/publicati on/262439895 Comparison of levels of_anxiety_and_depression_among_a ctive_and_sedentary_elderly/links/543 d0d1 $\bar{a} 0 \mathrm{cf} 20 \mathrm{af} 5 \mathrm{cfbf} 9 \overline{\mathrm{c}} 7$.pdf. 
14. Porcu M, Scantamburlo VM, Albrecht NR, Silva SP, Vallim FL, Araújo CR, et al. Estudo comparativo sobre a prevalência de sintomas depressivos em idosos hospitalizados, institucionalizados e residentes na comunidade. Medicina (Paraná) [periódico na internet]. 2002 [citado 2017 set. 11]; 21(3): 24(3):713-7. Disponível em: http://ojs.uem.br/ojs/ index.php/ActaSciHealthSci/article/vie $\mathrm{w} / 2498$

15. Oliveira MF, Bezerra VP, Silva AO, Alves MSCF, Moreira MASP, Caldas CP. Sintomatologia de depressão autorreferida por idosos que vivem em comunidade. Saúde Coletiva (Rio de Janeiro) [periódico na internet]. 2012 [citado 2017 ago. 25]; 17(8):2191-8. Disponível em: http:// www.redalyc.org/html/630/630230730 29/

16. Borges DT, Dalmolin BM. Depressão em idosos de uma comunidade assistida pela estratégia de saúde da família em Passo Fundo, RS. Medicina de Família e Comunidade (Florianopolis) [periódico na internet]. 2012 [citado 2017 set. 11]; 7(23): 7-14. Disponível em: https://rbmfc.org.br/rb $\mathrm{mfc} /$ article/view/381

17. Melo NCV, Ferreira MAM, Teixeira KMD. Condições de vida dos idosos no Brasil: uma análise a partir da renda e nível de escolaridade. Economia Doméstica (Viçosa) [periódico na internet]. 2014 [citado 2017 set. 15]; 25(1):004-19. Disponível em: http://www.seer.ufv.br/ seer/oikos/index.php/httpwwwseerufvb rseeroikos/article/view/154/182
18 Maximiano-Barreto MA, Montenegro AV, Fermoseli AFO. Qualificação profissional e impactos na Qualidade de vida: um estudo com idosos da comunidade Santo Onofre em Maceió - AL. Psicologia (Maceió) [periódico na internet]. 2017 [citado 2017 set. 22]; 4 (1): 47-56. Disponível em: https://periodicos.set.edu.br/index .php/fitshumanas/article/view/4055

19. Castro-Costa E, Dewey M, Stewart R, Banerjee S, Huppert F, MendoncaLima $C$, et al. Ascertaining late-life depressive symptoms in Europe: an evaluation of the survey version of the EURO-D scale in 10 nations. The SHARE project. Rev. Inter. Mét. Pes. Psiq. 2008; 17(1): 12-29. Disponível em:http://onlinelibrary.wiley.com/doi/ 10.1002/mpr.236/abstract

20. Santos WJ, Giacomin KC, Firmo JOA. Avaliação da tecnologia das relações de cuidado nos serviços em saúde: percepção dos idosos inseridos na Estratégia de Saúde da Família em Bambuí, Brasil. Saúde Coletiva (Belo Horizonte) [periódico na internet]. 2014 [citado 2017 set. 11]; 19 (8): 3441-50. Disponível em: http://www. redalyc.org/html/630/63031151015/

21. Ministério da Saúde (BR). Caderno de Atenção Básica. Envelhecimento e Saúde da Pessoa Idosa. Brasília: Ministério da Saúde; 2016.

22. Ministério da Saúde (BR). Política Nacional de Saúde da Pessoa Idosa. Portaria MS/GM n ${ }^{\circ} 2528$, de 20 de outubro de 2006 Brasília: Ministério da Saúde; 2006. 